\title{
Strategies for the management of Candida albicans infection
}

\author{
Vaishali, Johra Khan* and Wamik Azmi * \\ Department of Biotechnology, Himachal Pradesh University, Summerhill, Shimla-171005, Himachal Pradesh, India \\ *Department of Medical Laboratory Sciences, College of Applied Medical Sciences, Majmaah University, Al Majmaah, 11952, Saudi Arabia \\ *Health and Basic Sciences Research Center, Majmaah University, Al Majmaah, 11952, Saudi Arabia
}

\section{Article Info}

\section{Article history}

Received 6 October 2021

Revised 23 November 2021

Accepted 25 November 2021

Published Online 30 December 2021

\section{Keywords}

Candida albicans

Candidiasis

Antibiotics

Pathogens

Infection

Multidrug resistance

\begin{abstract}
Candida species are considered to be an opportunistic pathogen that leaves asymptomatically in warm blooded animals like human, birds etc. It can cause serious and lethal disease in healthy individuals. It generally causes infection of skin, oral cavity esophagus, gastrointestinal tract, vagina and vascular system of humans. It is a common cause of nosocomial infection in hospitals and most of the Candida infections occur in patients who are immunocompromised. Virulence factor like adhesion, morphogenesis, phospholipase are responsible for pathogenesis. Genus Candida include about 200 species but normally $C$. albicans, C. glabrata, C. tropicalis, C. parapsilosis, and C. krusei are five species of Candida involved in $90 \%$ of invasive infections. Candida infections can be superficial or invasive. Superficial infections often affect the skin or mucous membrane and can be treated successfully with antifungal drugs whereas invasive fungal infections are often life-threatening. Different chemical drugs such as azoles, polyenes, echinocandins and nucleoside analogues are in use to treat the infection caused by C. albicans, but due to their regular use, C. albicans can show resistance to these drugs and mortality rate increases. Resistance to various drugs is due to multiple factors, viz., reduced accumulations of drug in the cells, decreased target affinity and counteraction of the drug effect. Biofilms production is the another major cause of resistance to different drug groups and to overcome this emergence, different strategies are improvise which include the new triazoles, peptides, use of natural compounds, vaccinations, antibodies, cytokines therapy, and low level laser therapy.
\end{abstract}

\section{Introduction}

There are so many microbial infections in the world which may or may not be lethal. More than 7, 50,000 deaths recorded every year and the number will increase, if radial actions are not taken. Some common types of fungal infections are athlete's foot, yeast infection, onychomycosis. There are number of fungi are present but almost three hundred fungi are pathogenic to humans. Pathogenic fungus includes Candida albicans, Cryptococcus neoformanas, Candida glabrata, torula and many others. Almost $90 \%$ infections are caused by five spp. named as Candida glabrata, Candida tropicalis, Candida parapsilosis, Candida krusei and Candida albicans. Infections caused by $C$. albicans are of greater threat to humans. $C$. albicans mostly occur in the GI tract, mouth, and vagina, mucosal membrane, skin, oral pharyngeal of human and other warm blooded animals. C. albicans are also present in birds' digestive tract. Pathogenecity of $C$. albicans increases if the person is immunocompromised. C. albicans cause infection due to its overgrowth and cause of overgrowth can be high sugar level, weak immunity, use of medical equipment, malnutrition, neutropenia People taking medications like steroid or antibiotics, having removable dentures are more susceptible for candidiasis. When the growth increases toxic byproducts like acetaldehyde, ammonia and uric acid produced by $C$. albicans also increases and this leads to cause various changes in the body and body shows symptoms such

Corresponding author: Dr. Wamik Azmi

Professor, Department of Biotechnology, Himachal Pradesh University, Summerhill, Shimla-171005,Himachal Pradesh, India

E-mail: wamikazmi@rediffmail.com

Tel.: +91-177-2831948

Copyright () 2021 Ukaaz Publications. All rights reserved.

Email: ukaaz@yahoo.com; Website: www.ukaazpublications.com as fatigue, headache brain fog, illusion, digestive problems, nausea, vomiting, dizziness, etc., some uncommon symptoms may also appears including acid reflux, bloating, asthma, allergies, constipation, cracked tongue, skin rash, hallucination, indigestion, irritability, bloody stool, stiffness, insomnia, bleeding gums itching, etc.

\section{General characteristics of C. albicans}

C. albicans generally present in the mucosal membrane of human and other warm blooded animals, in the gut, the vagina, skin surface and the digestive tract of birds. It can be isolated from soil, food, animal, hospitals and in-animate objects. They are small in size around 2-4 um in diameter and oval shaped. They can be as yeast, pseudohyphae and hyphae. A component in human saliva is responsible for the conversion of fungal hyphae to budding yeast. C. albicans are gram-positive, encapsulated, diploid, spore forming and polymorphic fungus. The albicans spp. appears white when they are cultured and the spore formed are known as chlamydospore and these spores play important role in the unfavorable conditions like dry or hot seasons.

They are capable to form biofilms and the reproduction in C. albicans is by budding in which bud or a protoplasmic protrusion from mother cell is produced, grow and finally detach and form a new cell. Blastophore phase (reproduce by budding) and hyphal phase are two different phase in which $C$. albicans can grow. C. albicans is commensally harmless but opportunistic pathogen and exist in the GI tract (Calderone et al., 2009). Approximately $75 \%$ of women's suffer from Candida infection once in their lifetime. C. albicans generally found in the human gut flora but it can survive outside the human body. Around 50\% of healthy adults carry it in 
their gastrointestinal tract and mouth. It is referred as dimorphic fungus as it can grow either as yeast or filamentous cell and hyphal form is more invasive as compared to yeast form.

Candida is found worldwide but persons with deficiency in immune system such as HIV and cancer are more susceptible for $C$. albicans infection. Patients in intensive care units and undergone surgery are at higher risk. Transmission can be through mother to infant, people to people in different areas (especially hospitals), from healthcare workers, sexual contact with woman suffered from vaginal yeast infection. C. albicans spp. cause infection mainly in four stages: colonization, superficial infection, deep seated infection, dissemination infection. It commonly occurs as superficial infection which involves epithelial penetration and degradation of host protein.

The $C$. albicans biofilms develop in a sequential manner which involves adhesion of microorganism to the surface, colony formation and organization of cell, secretion of extracellular polymeric substances formation of 3D structure and dispersion of cell (Muhleschlegal and De Sordi 2009). On the basis of its pathogenesis, C. albicans can cause many type of infections and these infections are called as candidiasis. Candidiasis mainly divided into two major groups; one group is mucosal infection and the example is oral thrush which appears as white rashes on body. Generally affected areas are vagina, oropharyngeal mucosa and gastrointestinal epithelial cells. In women mostly, the infected area is vagina and the infection is known as vulvo vaginal candidiasis and if this infection appear again and again then called as recurrent vulvo vaginal candidiasis. Another group of infection is systematic infection which are the life threatening having death rate of about $30 \%$. This type of infection is commonly seen in the person having transfer plantation immunocompromised chemotherapy or low birth weight infants.

The $C$. albicans are most pathogenic among all the spp. of Candida. This is due to various virulence factors involved including its morphological transition. As it can transist between yeast and hyphal forms. Other reasons include the adhesion and invasion of the cell surface, biofilm formation and secretion of hydrolytic enzymes. At $\mathrm{pH}$ level $6, C$. albicans grows as yeast while at $\mathrm{pH}$ level 7 , it acts as hyphal growth. Including this starvation, presence of serum, physiological temperature, and $\mathrm{CO}_{2}$ are also responsible for the hyphal growth.

Most common type of Candida infections are urinary tract infection (UTI), genital yeast infections, oral thrush, mucocutaneous candidiasis, Candida pulmonary infection. These infections can be diagnosed by different clinical methods.

Microscopic methods are used to examine $C$. albicans spp. in the sample. If , the person is infected with candidiasis, antifungal agents are used to treat the disease. Polyenes, azoles, echinocandins and nucleosides are the antifungal groups which are used to treat candidiasis. Major representative of polyenes are amphotericin B and nystatin. Azoles include fluconazoles, itraconazole, and imidazoles. Echinocandins include anidulafungin, caspofungin and micofungin. Flucytosine is an example of nucleoside analogues. But, C. albicans spp. can show resistance to these chemical groups of drugs due to their use for the long time. To overcome this problem, new strategies are developed to fight with candidiasis.

\section{Infection caused by $C$. albicans}

The $C$. albicans present in various body parts of the human as part of natural microflora but they cause disease when they over grow due to long course of antibiotics or weakened immune system (Pappas et al., 2015). Most common types of Candida infections are urinary tract infection that is UTIs, genital yeast infection, oral thrush, mucocutaneous candidiasis, Candida pulmonary infection, Beside these infections Candida can also cause some other disease such as neutropenia, candidemia, endocarditis, endophthalmitis, meningitis, intra abdominal candidiasis, osteomylitis, and fungal arthritis.

\subsection{Urinary tract infection}

UTIs it is also called as urinary tract candidiasis. It can be caused by Candida spp. but most common cause is $C$. albicans. Urinary tract candidiasis is the most frequent nosocomial fungal infection. It occurs in the urinary tract but severe diseases are found when it spread up to the kidneys (Cernicka and Subik, 2006). Men are at lower risk of developing UTIs then the women. People undergoing a course of antibiotics, diabetic, having any medical instruments such as urinary catheters inserted in body having a weakened immune system, hospitalized for long time or in the ICUs are at higher risk of urinary tract candidiasis (UTIs).

People suffering from UTIs may experience pain in the pelvic area, bladder, lower abdomen and groin. Pain during urination, continue impulse to urinate with burning sensation, urine color changes and become darker having foul smell and blood in urine has seen in the patient. If infection spread to the kidney, then the symptoms may be back pain, vomiting, fever, and nausea (Table 1). If a patient is symptomatic and found infected with $C$. albicans fluconazole is the antifungal drug used for UTIs.

\subsection{Genital yeast infection}

Genital yeast infections are caused due to the overgrowth of $C$. albicans in the genital area. It is the infection of vagina and vulva. When Lactobacillus level in the genital area decreases the number of $C$. albicans spp. increases because lactobacilli are the reason for the controlled growth of $C$. albicans. It is caused by Candida spp. so also called as vaginal candidiasis. It is mostly occur in women and infect the area of vagina and the infection is known as vulvo vaginal candidiasis and if the infection appear again and again then it is called as recurrent vulvo vaginal candidiasis. Vaginal yeast infections cannot be transmitted sexually but may appear after first sexual activity (Riley et al., 1986). Genital yeast infections can be established after engaging in certain sexual activities like involvement of oral-genital contact. Pregnant women, diabetic people, people on hormone therapy, people having impaired immune system, women with high estrogen level are more susceptible for genital yeast infections.

Genital yeast infections are of two types: mild or moderate and severe type of genital infection. Moderate infections can be cured by the short duration courses of OTC and single dose of an oral antifungal medication like fluconazole can be used, whereas for severe diseases long time courses are prescribed. Antifungal agents are used in the form of creams, pills, or ointment. Precautions should be taken and personal hygiene is the best precaution method to prevent candidiasis. 


\subsection{Oral thrush}

Oral thrush is also called as mouth thrush and oral candidiasis. Oral thrush is infection of mouth due to the overgrowth of $C$. albicans which are a part of our normal flora. Oral thrush infection is not only limited to the mouth but also affect the tonsils and the throat as well. Severe infection of oral thrush may affect the esophagus also. It is commonly found in the baby but people with impaired immune system and who use steroid sprays, people who wear dentures are also at high risk. Chemotherapy, radiation therapy can also kill the normal cells of the body and make a person more susceptible to oral thrush. It is a short term disease which can be resolved within days to weeks and it can be diagnosed easily. White patches on the tongue are most common symptom of oral thrush. Infected area may be swallow and person can feel pain, redness in the infected area, loss of taste, burning sensation, plaque formation are other symptoms (Table 1). Oral thrush can be spread by kissing and infection may pass through one body part to another in the patient itself or from one part of the body to another part of someone else body.

Antifungal drugs like miconazole, clotrimazole, econazole are given as creams or pills to clear the skin infection. Home remedies are also present for the treatment of oral thrush. Mouthwash or mouth sprays should be avoided to prevent oral thrush. To prevent the swelling and itching of the infected area, steroid creams are used. Fluconazole pills are given in the severe infection and they are prescribed orally. Oral thrush can be converted to systematic infections if not treated, especially in the case of weakened immune system.

\subsection{Mucocutaneous candidiasis}

Mucocutaneous candidiasis is the rare genetic infection caused by C. albicans generally occurs at mucosal, nail or skin surfaces. People having genetic defects or with impaired immunity system are more susceptible to mucocutaneous candidiasis, and persisting for long time or constantly recurring and known as chronic mucocutaneous candidiasis (Eye rich et al., 2010). The main reason for mucocutaneous candidiasis may be wearing synthetic undergarments and poor hygienic conditions. On the site of infection, red rashes are formed. Lesion formations on the infected area provide evidences for mucocutaneous candidiasis (Huppler et al., 2012). On the skin, white curd like substance is produced. Symptoms of mucocutaneous candidiasis vary from mild to severe (Table 1). Mutations that cause immunodeficiency are diagnosed by blood tests and sample taken is examined under microscope to check the presence of $C$. albicans. To cure skin infections, antifungal creams are best treatment. Antifungal drugs like clotrimazole, econazole, can be used. To avoid swelling and itching in the infected area, steroid creams can be used. Oral fluconazole pills are used in case of widespread. Preventive measures like good hygienic conditions and avoiding smoking or alcohol can be great help to reduce the cases of Candida infections.

\subsection{Pulmonary disease caused by Candida}

Candida spp. has been seen in the sputum and the range of Candida spp. in the sputum varies between 3 to $84 \%$ depending upon the method of isolation and sample taken. It is also called as pulmonary candidiasis. It has been seen mostly in the immunosuppressive patients. Infection entered the lungs by aspiration and hematogenus spread. Candida albicans are not likely to cause pulmonary candidiasis, but if a person has acquired pulmonary candidiasis, the patient is ill from multiple other diseases. Three type of pulmonary candidiasis are there include embolic pulmonary candidiasis, disseminated pulmonary candidiasis, and bronchopulmonary candidiasis. Amphotericin B is commonly used to treat pulmonary candidiasis. Fluconazole, itraconazole, ketoconazole and imidazoles are other alternates to fight with candidiasis successfully.

Table 1: Candida infections and their treatments

\begin{tabular}{|l|l|l|}
\hline Type of Candida infection & \multicolumn{1}{|c|}{ Symptoms } & \multicolumn{1}{c|}{ Treatments } \\
\hline Urinary yeast infection & $\begin{array}{l}\text { Pelvic pain, blood in urine, burning sensation when } \\
\text { urinating, increased need to urinate. } \\
\text { Genital yeast infection }\end{array}$ & $\begin{array}{l}\text { Fluconazole. } \\
\text { rash around the vagina, redness or swelling around } \\
\text { vagina. }\end{array}$ \\
Oral thrush & $\begin{array}{l}\text { Loss of taste, swallowing, redness inside the mouth, } \\
\text { white spots in the mouth, burning sensation in mouth. } \\
\text { Thickened skin, lesions can form on body, } \\
\text { appearance of white curd like substance on } \\
\text { skin. }\end{array}$ & $\begin{array}{l}\text { Nystatin and clotrimazole in form of pill } \\
\text { or liquid. } \\
\text { ointment }\end{array}$ \\
\hline
\end{tabular}

Besides these, some other types of infections are also are caused by $C$. albicans.

\subsubsection{Neutropenia}

Neutropenia is the condition when number of neutrophils decrease. Neutrophils are the guard of human body which attacks the microorganism when they invaded the body. Neutropenia is not caused by Candida but due to neutropenia, C. albicans can easily attack the body and infection may occur. Infection due to neutropenia can occur as ulcer, rash, wounds that are not healing, fever. Neutropenia can be caused by several factors like problem in the bone marrow, nutritional deficiency, and other infection.

\subsubsection{Candidemia}

Candidemia is a fungal infection caused by $C$. albicans in the bloodstream and $10 \%$ of bloodstream infections are caused by Candida. Chances of candidemia increases if the patient is admitted in the intensive care unit (ICU) for prolong period or use any device like catheter. Risk of candidemia is more in the people who are suffering from immunosuppression, going through surgery, or using antibodies for a long period. The candidemia is mainly caused by $C$. albicans but some other causative agents are $C$. tropicalis, $C$. glabrata C. parapsilosis. In case of children, C. parapsilosis is the 
causative agent most of the time (Colombo et al., 2006). Once candidemia is a suspected, treatment should be started as it is a lifethreatening disease. Numbers of medications are available for the Candida infection. Some of them are fluconazole, amphotericin B, caspofungin and micofungin. The selction of medication depends on the severity of illness and species of Candida which acts as a causative agent.

\subsubsection{Endocarditis}

It is the infection in the inner lining of heart called as endocardium and the main symptom of endocarditis is swelling and redness in the endocardium. Endocarditis is caused by microorganisms which are present in the body part like mouth and spread through bloodstream and reaches heart where they are fixed to the damaged part of the heart. People who are suffering from heart disease having damaged heart valves or artificial valve are at higher risk to be suspected with endocarditis. Besides this, people with weak immune system using antibiotics for long time, going through a surgery or using medical devices such as food pipe, catheter, heart valve improper healthcare, etc., are also so at a greater risk. If a person is suffering from endocarditis, he or she may also suffered with blood clotting, kidney damage, enlargement in spleen, abscesses in the body parts like heart, brain, lungs. Fluconazole and amphotericin B are used to treat endocarditis (Durack, 1995).

\subsubsection{Endophthalmitis}

It is the infection of the eyeball and mainly fluid or the tissues get infected. It is a severe disease because a person can be blind if this is not treated sooner. Exogenous and endophthalmitis and endogenous endophthalmitis are the two main types of the endophthalmitis. Exogenous endophthalmitis as per its name, the causative agent of the endophthalmitis comes from the outside the body due to many reasons including eye injury. Exogenous endophthalmitis are of two types, i.e., chronic and acute. Chronic endophthalmitis show symptoms in its earlier stage means within few days of getting infection whereas in case of acute endophthalmitis, symptoms begin after a longer time. Endogenous endophthalmitis is due to the infection caused within the body which will spread to the eye. C. albicans are the main fungal causative agent of endophthalmitis but sometime $C$. tropicalis can act as a causative agent. Antibiotics are the main courses for treatment and steroids are used to decrease the swelling.

\subsubsection{Meningitis}

It is the inflammatory infection in the tissue of brain and spinal cord surrounding called meninges. Meningitis is really caused by fungal agent and major causative agent for meningitis is viruses. Fungal meningitis can be caused by Cryptococcus, Histoplasma, Blastomyces, Coccidiodes and Candida. Amphotericin B and fucocytospin are used to treat meningitis caused by $C$. albicans.

\subsubsection{Intra-abdominal Candidiasis}

It is also termed as Candida peritonitis which is the inflammation to the abdominal lining. The major causative agent is $C$. albicans but other Candida spp. may be involved. Fluconazole, amphotericin $\mathrm{B}$ and caspofungin micofungin may be used for the recovery purposes.

\subsubsection{Osteomylitis and fungal arthritis}

Osteomylitis is the infection condition which leads to the irritation in the bones of legs, arms or spine and fungal arthritis is a condition in which joints of bones get swallowed and inflammation may occur. It can be referred as mycotic arthritis and C. albicans are infection is responsible for these conditions. Amphotericin B and aluconazole are antifungal drugs used to cure the disease.

\section{Treatments available for infection caused by $C$. albicans}

The disease caused by $C$. albicans ranges from non-serious disease to life threatening disease and it can involve any part of the body (Blumberg et al., 2001; Kuhn et al., 2002). Most of the cases of candidiasis are due to biofilms formation on the site of infection that maybe mucosal and systematic (Jabra-Rizk et al., 2004). To fight with this disease, antifungal agents are used as the treatment. Polyenes (inhibit the growth of pathogen), azoles (kill the pathogen), echinocandins (inhibit the growth of pathogen), and nucleosides (inhibit the growth of pathogen) are the licensed antifungal group (Table 2) for the treatment against Candida infections (Veerdonk et al., 2010; Ruhnke et al., 2011; Arendrup et al., 2017; Oliveira et al., 2018).

\subsection{Polyenes}

Amphotericin B and nystatin are the example of polyene antifungal agents. Polyene works on the fungal cell membrane ergosterol and cause porosity and finally leads to cytoplasmic loss of the cell (Henry et al., 2000; Onyewu and Heitmann, 2007; Williams et al., 2010). The another method involved for the treatment is interaction with lipoprotein after the oxidation-reduction reaction that ultimately diminish the membrane permeability due to deliverance of free radicals (Barwicz et al., 1998; Onyewu and Heitmann, 2007). The nystatin was the first drug for treatment and control of cutaneous and mucocutaneous candidiasis and it is available in the form of creams and oral washes. It is a fermentation product Streptomyces noursei and listed in the essential medicines by WHO. Naytatin binds to fungal sterol that is ergosterol which leads to a $\mathrm{K}^{+}$leakage and ultimately death of fungus. Since, ergosterol is unique molecule in the fungus, hence, nystatin does not cause any adverse effects to human or plant when injected in a proper composition (Groll, 1997). Some other use of nystatin include in the treatment of skin, vaginal, mouth infections caused by Candida. Oral candidiasis due to mouth dentures can be treated with nystatin and it can also be used in infants to prevent invasive fungal infections.

Another example of polyene is amphotericin B which has widely been used to treat disease such as mucormycosis, aspergillosis blastomycosis and cryptococcosis. As amphotericin B has poor oral and intramuscular absorption, it is used with a colloidal suspension in deocycholate or liposomal amphotericin B and amphotericin B colloidal dispersion (Ruhnke et al., 2011). The liposomal amphotericin $\mathrm{B}$ can be used as an antifungal to immunocompromised patients. It is highly effective drug with low incidence of drug resistance. The only chance of Candida resistance to polyene is when the ergosterol content of cell membrane is low (Sanglard et al., 1996; Williams et al., 2010).

\subsection{Azoles}

These are the antifungal agents which act on the P450-dependent enzyme lanosterol $14 \alpha$-demethylase, which plays an important role in the production of ergosterol. This interference ultimately 
leads to the accumulation of sterol intermediates and ergosterol level in the fungal cell membrane gets depleted (Bossche et al., 1987; Henry et al., 2000; Onyewu and Heitmann, 2007). Azoles also inhibit mammalian cytochrome-P450 enzymes and lanosterol converted into cholesterol which inhibit the synthesis of ergosterol. Azoles are categorized into two types: imidazoles and triazoles. Imidazoles is used for the systematic treatment of mycosis while terconazole used for the treatment of vaginal candidiasis. The clotrimazole, econazole and miconazole are also used to treat vaginal candidiasis. Imidazoles used in case of Candida infection of mouth, vagina, and skin are active against many Candida infections but they are inactive against $C$. krusei. Fluconazole can be used to treat infections from non-albicans Candida spp. and it has successive rates to vaginal, mucosal, dermal and systematic candidiasis (Franz et al., 1998; Safdar et al., 2001). Itraconazole can be used in case of a patient who show non-responsiveness to fluconazole for the treatment of oral and oesophageal candidiasis (Johnson et al., 1995). Voriconazole is another triazoles which can be used as the alternative to fluconazole and itraconazole. It is also safe for the patients having severe diseases like AIDS or cancer.

\subsection{Echinocandins}

They are semisynthetic antifungal lipopeptides in which lipid side chains are linked to the centre that is cyclic hexapeptide (Onyewu and Heitmann, 2007). It works on inhibition of $\beta-1,3$ - D- glucan synthase. Glucant is a polysaccharide component of cell wall of pathogenic fungi, which maintains osmotic integrity and help in cell division and growth. Anidulafungin is a type of echinocandins used to treat Candida infection in the blood or in the stomach or esophagus as an alternative treatment for oropharyngeal candidiasis. Caspofungin is a lipopeptide antifungal drug found to be more effective than fluconazole and Amphotericin B and can be used to treat disease caused by aspergillus and Candida spp. Micofungin is used to treat candidemia and other Candida infections and it inhibits the synthesis of $\beta-1,3$ glucan.

\subsection{Nucleoside analogues}

Nucleoside analogues are composed of flucytosine and used to treat severe fungal disease throughout the body like blood, lungs, heart, urinary tract caused by species like Candida or Cryptococcus. It can be used in combination with amphotericin B for treatment of diseases like septicemia, endocarditis, urinary tract infection, meningitis and pulmonary infection. Mechanism of action of flucytosine is not exactly known, but it has been proposed that this drug enters to the fungal cell via cytosine permease and metabolizes itself into 5-fluorouracil and 5-fluorodeoxyuridine phosphates. The 5-fluorouracil reacts with fungal RNA and stops the synthesis of DNA and RNA and ultimately leads to death of organisms.

The allylamines and thiocarbamates are other drugs which disrupt the cell membrane by inhibiting the squalene-epoxidase, an enzyme important for the synthesis of ergosterol (Sanglard et al., 1996).

Table 2: Classes of antifungal agents and their mechanism of action

\begin{tabular}{|l|l|l|}
\hline Classes & \multicolumn{1}{|c|}{ Mechanism of action } & \multicolumn{1}{c|}{ Examples } \\
\hline Polyenes & $\begin{array}{l}\text { Binds to ergosterol in fungal cell membrane and interaction } \\
\text { with lipoproteins after oxidation-reduction reaction. } \\
\text { Anhibition of lanosterol 14 } \alpha \text {-demethylase. It leads to depletion } \\
\text { of fungal cell membrane ergosterol level. } \\
\text { Inhibition of } \beta-1,3 \text {-glucan synthase. }\end{array}$ & $\begin{array}{l}\text { Nystatin, Amphotericin B } \\
\text { Fluconazole, Itraconazole, Miconazole, } \\
\text { Voriconazole, Ketoconazole, Posaconazole, } \\
\text { Caspofungin, Micofungin, Anidulafungin }\end{array}$ \\
Fucleoside analogues & Pyrimidine analog that inhibit DNA and RNA synthesis. & Flucytosine \\
\hline
\end{tabular}

\section{Mechanism of multidrug resistance of $C$. albicans}

There are variety of antifungal drugs to cure infection caused by can C. albicans. However, C. albicans pathogen started showing resistance toward these drugs. The multidrug resistance can be defined as resistance of single spp. toward more than one drug or acquired non-susceptibility to at least one agent in three or more antimicrobial agents. The intrinsic resistance is the resistance when spp. are naturally resistance to a antibiotic and acquired resistance is a type of resistance in which species obtain the ability to resist the activity of antimicrobial agent to which it was susceptible. Acquired resistance is obtained by various mechanisms but easiest way is mutation. The resistance to the drug ultimately leads to high morbidity and mortality rate in patients especially patients with impaired immunity (Morace et al., 2014). Resistance to the different antifungal drugs have different mechanism of action but there are three basic resistance mechanisms responsible, which include reduced intracellular accumulation of drug, decreased target affinity and counteraction of the drug effect (Spampinato and Leonardi, 2013).

\subsection{Resistance to polyenes}

Polyenes are used to treat Candida infection for last few decades and amphotericin B, nystatin and natamycin are some example of widely used polyenes. C. albicans shows resistance to amphotericin B whereas some other species of $C$. krusei and C. glabrata show susceptibility to this drug. Higher doses of drug are prescribed to treat the infection caused by resistant Candida spp. (Rex et al., 2000). The antifungal lipopeptide caspofungin may be responsible for the drug resistance in the patients undergone any transplants (Krogh-Madsen et al., 2006). General mechanism of action of polyenes depends on the destruction of fungal cell membrane. Polyenes directly interact with ergosterol synthesis and creates pores in the cell membrane and release of cellular component leads to the death of the fungal cell (Ellis et al., 2002).

\subsubsection{Resistance to polyene is due to counteraction of drug effect mechanism}

Polyenes interact with the ergosterol to treat infection and the interaction rate of polyene to ergosterol is highest, if compared with other type of sterols like exosterol. Acquired resistance in the polyene is result of reduction in exosterol content in the cell wall composition (Table 3). Important point to note is that Candida spp. which are resistant to polyene has decreased amount of ergosterol. However, in acquired resistance, the favored component ergosterol is replaced by others sterols (lanosterol, fecosterol, 
lichesterol and episterol ) which are not easily targeted by the polyene. The change in the composition of fungal cell wall is because of mutation in the ERG3 and ERG6 genes responsible for the biosynthesis of ergosterol. These mutations lead to production of others sterols which are not targeted by polyenes. The decrease in the ergosterol content and increase in the sterol component in the cell membrane of fungal cell will lead to resistance to polyenes.

Another mechanism of resistance to polyene is related to various stages of growth of fungal cell. The log phase of cell growth involve the breakdown and re-synthesis of cell wall at higher rate, which helps the amphotericin B to reach the cell membrane of fungal cell. However, in stationary phase of cell growth, break down and resynthesis process slow down and amphotericin B is not able to access the cell membrane easily and this makes fungal cells resistant to polyene due to its inability to follow its general protocol to rupture the cell wall of fungal cell (Rex et al., 2000). Candida spp. other than $C$. albicans shows resistance or susceptibility according to their capability of mutation. C. glabrata and C. parapsilosis are highly resistance because they can mutate easily due to their haploid nature. C. glabrata are more resistance to resistant to polyenes than C. albicans (Odds et al., 1987).

\subsection{Azole resistance}

Itraconazole and fluconazole are azoles widely used for the treatment of candidiasis especially in the immunocompromised patients because it is safe and can be taken orally due to its water solubility (Meris and Verweji, 2000; Hoffman et al., 2000; Livermore, 2004). The fluconazole resistant cases were reported mostly in HIV patients suffering with oropharyngeal candidiasis (Redding et al., 2003; Skiest et al., 2007). However, azoles resistance are uncommon in case of vaginal diseases and candidemia (Ribeiro et $a l ., 2005)$. The other species of Candida like C. glabrata, C. krusei and C. lastiniae were also reported to be resistant to fluconazole (Vazquez et al., 2001; Pfaller et al., 2004; Pfaller et al., 2007).

\subsubsection{Reduced drug intracellular concentration/expression of efflux pump}

The intracellular concentration of azoles decreases due to over expression of efflux pump. An efflux pump belongs to two different classes. One of the classes of a flux pump is ATP binding cassette (ABC) transporters (Cannon et al., 2009) and other class is named as major facilitators superfamily (MFS). The two classes are different from each other on the basis of energy source, they used to pump out the drug and specificity of the azole molecule. The flux pump mechanism is regulated by several resistance genes and resistance of $C$. albicans to various azole drugs increase when the mRNA level of CDR1, CDR2, CDR3 and CDR4 increase (Marichal et al., 1997; Marr and Lyons, 1998).

\subsubsection{Decrease target affinity for the drugs}

Azoles are the antifungal drugs which act on the enzyme (generally encoded as ERG11), essential for the synthesis of ergosterol. Any type of mutation in the ERG11 gene or altered pathway of synthesis will ultimately leads to the resistance of azole antifungal drugs (Rogers et al., 2003). Upregulation of ERG11 due to these mutations result into increased synthesis of enzyme lanosterol $\alpha$ demethylase which is a key enzyme for the synthesis of ergosterol. This increased synthesis of ergosterol overcomes the antifungal drug activities. Azole resistance due to decreased target of affinity is not only seen in the C. albicans but also in the non-albicans spp. like C. glabrata, and $C$. dubliniensis (Perea et al., 2002). Main mechanism behind reduced binding between azoles and the target is the point mutation in ERF11 gene, which results into the conformational changes (White et al., 1998). Mutations in the structure occur in many different ways.

\subsubsection{Counteraction of the drug effect}

Counteraction of the drug effect by increasing the production of ergosterol with the help of ERG1 1 gene simply increases the number of target proteins. This increase in target protein lead to less susceptibility of fungal cell to the antifungal drug. Another mechanism involve the alteration in the process of synthesis of ergosterol by inactivating ERG3, responsible for the activation of 5-esterol deasturase which convert episterol to ergosterol.

\subsection{Echinocandin resistance}

Most of the infections caused by Candida spp. are due to the biofilms production. Biofilms are group of $\mathrm{m} / \mathrm{o}$ forming three dimensional structures on biotic and abiotic surfaces. These biofilms show natural resistance to the antifungal drugs like azoles, and amphotericin B which are widely used to treat Candida infections. In the recent years, resistance to azoles has seen in the $C$. albicans. Due to this reason echinocandins, a group of antifungal agents come to knowledge as an alternative for azoles. Echinocandin drugs are prescribed as first line therapy in case of invasive candidiasis. Echinocandins are lipopeptidic antifungal which interfere in the glucan synthesis. Echinocandins interact with the enzyme $\beta-(1,3)$ D-glucan synthase leads to inhibition in the synthesis of $\beta-(1,3)$ $D$-glucan due to this a weak fungal cell wall is formed and can result to osmotic lysis and ultimately cell death. Further, not only $C$. albicans but also non- albicans spp. of Candida such as C. krusei, $C$. glabrata, and $C$. tropicalis show resistance to echinocandins (Krogh-Madsen et al., 2006: Pasqualotto et al., 2008). Resistance to echinocandins is mostly seen in C. glabrata. Resistance in echinocandin is achieved by various mechanisms one of them is prolonged use of echinocandins in case of Candida infections. Cases of resistance are mostly reported in the immunocompromised patients and infection rapidly spread in the area of hospitals. Main mechanism of resistance to echinocandin is decreased target processivity for the drugs.

This mechanism is mainly based on the mutation. Mutations generally occur in two genes, named as FKS1 and FKS2 gene (Park et al., 2005; Balashov et al., 2006). These two regions are known as hot spot regions (Table 3). In maximum cases of resistance to echinocandins only a single mutation is responsible for the resistance but there are some cases also found in which resistance is due to change in more than one gene. FKS1 and FKS2 genes are responsible for the synthesis of $\beta(1,3) \mathrm{D}$-glucan synthase and fungal activity is inhibited by inhibiting the synthesis of $\beta(1,3)$ D-glucan. So, mutations in these regions lead to resistance for echinocandins. Mutations in either FKS1 or FKS2 genes at first or fifth position (phenylaline and serine, respectively) cause increase in MIC level. Mutations in FKS region of $C$. albicans are not related to binding affinity but cause decrease in the $\mathrm{V}_{\max }$ values (Alexander et al., 2013). Main cause of echinocandin resistance is decrease in synthesis of glucan and elevated MIC value (Hernandez et al., 2004). Some Candida spp. may be natural resistant to echinocandins as they have natural polymorphism in FKS genes. 


\subsection{Flucytosine resistance}

Flucytosine resistance can be obtained by two resistance methods: primary resistance method and secondary resistance method. Flucytosine is a fluorinated pyrimidine which enters into the cell through cytosine permease and then drug 5-flucytosine converts into a metabolic active compound 5-fluorouracil. This metabolic active compound reacts with fungal RNA and inhibits transcription and protein synthesis. Flucytosine changes into fluorouracil which is a toxic compound.

Flucytosine resistance is primary when the resistance develops due to decreased uptake of the drug by cytosine permease whereas; secondary resistance is related to inactivation of enzyme which plays role in the pyrimidine pathways. Secondary resistance can be result of two main mechanisms that are reduced drug intracellular accumulations and counteraction of the drug effect. Reduced drug intracellular accumulation is due to mutations in FCY2 gene which is a code for cytosine permease (Table 3 ). As cytosine permease is mutated flucytosine, it is not able to enter the cell wall and for processing of metabolic activities (Peman et al., 2009; EspinelIngroff et al., 2016).

Second mechanism involves mutations in the FCY1 gene and FUR1 gene which encodes cytosine deaminase and uracil phosphoriblosyl transferases, respectively. Conversion of 5-flucytosine to 5fluorouridine monophosphate is reduced due to mutations. This type mechanism of resistance is mostly seen in the case of $C$. albicans but also in other non-albican spp. acquired resistance to flucytosine is maximum times due to point mutation in the FCY1 and FUR1 genes but several other mutations are also described in case of C. albicans, and C. glabrata (Kabir et al., 2012). The $10 \%$ primary isolates are found resistant to flucytosine when used with amphotericin B. It has been observed that the resistant to flucytosine is mainly by inhibiting pyrimidine biosynthesis which is achieved by inhibition of conversion of 5-flucytosine to 5-fluorouracil triphosphate and finally to 5-fluorodeoxyuridine monophosphate (Hakki et al., 2006; Duval et al., 2016; Kahn et al., 2007).

Table 3: Mechanism of resistance to various drugs

\begin{tabular}{|l|l|}
\hline Group of drugs & \multicolumn{1}{c|}{ Mechanism of resistance } \\
\hline Polyenes & $\begin{array}{l}\text { Alteration in ERG3 and ERG6 genes to inhibit the synthesis of ergosterol. } \\
\text { Reduced drug intracellular conc. By ABC transporters and MFS. Decreased target affinity of the drugs by } \\
\text { alteration in ERG11 gene.Counteraction of drug effects. } \\
\text { Mutation in the hot spot region. FKS1 and FKS2 are the hotspot regions. Mutation in either FKS1 or FKS2. } \\
\text { Echinocandins }\end{array}$ \\
$\begin{array}{l}\text { Primary or secondary mechanisms. Decreased uptake of drugs by cytosine permease muation in FCY1 gene } \\
\text { and FUR1 genes. }\end{array}$ \\
\hline
\end{tabular}

\section{Novel strategies to fight Candida species infection}

Different chemical drugs are used to treat $C$. albicans infection but in the recent year, resistance against these drugs have been shown and this emergence of resistance is due to the excessive use of antimicrobials. As Candida spp. become resistant to the antifungal drugs, the mortality and morbidity rate of Candida infection increases drastically over the past two decades. So, noble strategies are the need of time to improve the outcome of patients suffering from Candida and the alternative should be more tolerant and efficient. Different types of alternative are present which includes use of new triazoles, peptides as antifungal agents, and plants as a source of anti-Candida natural compound, vaccination, antibodies, cytokines therapy, low level laser therapy, adaptive transfer of primary immune cell, photodynamic therapy etc. and these different strategies are able to decrease the morbidity and mortality rate due to the Candida infection (Rodrigues et al., 2014).

\subsection{Triazoles as alternative drugs}

Ravuconazole, albaconazole, isavuconazole, are some triazoles drugs which are the alternatives to the drugs available. These drugs are less toxic and provide immunity in the case when widely used drugs like fluconazole and voriconazole are resistant. Albaconazole are antifungal agent having mechanism of action depending on inhibition ergosterol synthesis. These have low toxicity value and greater tolerability, display greater activity against Candida spp. It shows better susceptibility than fluconazole. In case of vaginal candidiasis concentration of single dose of albaconazole is four times less than that of fluconazole (Pasqualotto and Denning, 2008).
A second generation triazoles named is isavuconazole acts as antifungal agent and shown better susceptibility than the drugs used commonly like amphotericin B, fluconazole, flucytosine, itraconazole, and voriconazole. Isavuconazole is effective against candidemia and invasive candidiasis. Principle of working is similar to the triazoles. Another drug having similar structure to isavuconazole is ravuconazole. It shows greater susceptibility in patients who show resistance to fluconazole. But the main drawback of isavuconazole is that it is required in the higher amount/ concentration to the patients who are fluconazole resistant as compared to fluconazole susceptible patients.

\subsection{Peptides as antifungal agents}

From various parts of body peptides are isolated which shows antimicrobial activity and these peptides are helpful to prevent different fungal infections. The main mechanism of action of these peptides depend on that they reduce the passage of substances from the fungal cell membrane which ultimately leads to the permeabilization. Different peptides isolated from human body include lysozyme, lactoferrin, defensin, histatin, and cathericidins. Lysozyme is an enzyme isolated from the saliva and respiratory secretions. In the early era, lysozyme is known for its antibacterial activity but now it has been observed that lysozyme is also active against different spp. of Candida, Penicillium and Aspergillus especially Aspergillus fumigatus. Mechanism of action of lysozyme is not exactly known but most likely it acts by reducing secreted aspartic protease activity. 
Lactoferrin is found in the different secretion from body, but it is mainly present in the saliva. It provides treatment against infection caused by C. albicans and C. krusei (Samaranayake et al., 1997). Production of cationic peptide which is responsible for antimicrobial activity is the main way of lactoferrin to fight with Candida infections (Oris, 2004). Hlf 1-11 is the first cationic domain of lactoferrin $\mathrm{h}$ which is released by pepsinolysis has higher antifungal activity (Lupetti et al., 2007). This antifungal activity is due to its capability of enhancing the production of macrophages and dendritic cells (Vander et al., 2012). Main advantage of lactoferrin is that it is able to inhibit the formation of biofilms in its early stages. This inhibition is due to reason that it becomes the cause of lower number of receptors on the surface of fungal cell and by interfering with biofilms cellular density and its metabolic activity (Morici et al., 2016).

Member of defensin family named as human $\beta$ defensin (HBD) are cationic peptides. Mainly three types of HBD show antifungal activity against $C$. albicans (Krishnakumari et al., 2009; Seleem et al., 2017; Tomalka et al., 2015). These three HBD include human $\beta$ defensin 1(HBD 1), human $\beta$ defensin 2 (HBD2) and human $\beta$ defensin 3 (HBD3). HBD-1 reacts when pro-inflammatory molecules like interferon- $¥$, a bacterium, lipopolysaccharides, etc., binds to TLR (Duits et al., 2002). HBD-1 is generally important in the mucosal Candida infections. HBD-1 is helpful in expression of HBD-2 (Tomalka et al., 2015). Bacteria and fungi when interact with TLR2 in epithelial tissue that enhance the expression of HBD-2. Then TGF- $\alpha$ binds to its receptor EGFR (epidermal growth factor receptor) the activity of HBD- 3 increases. Among the three HBDs, HBD- 3 is highly active against various fungi (Krishnakumari et al., 2009) and these three mechanisms are involved in the cell permeability which leads to cell death (Krishnakumari et al., 2009). But, some important factors of these peptides are not known and still require research on some of these like its hemolytic activity, their production modes, how they interact with low salt concentration, and the violence activity. These factors could limit their use in the clinics.

\subsection{Plant as a source of anti-Candida natural compound}

Plant and its molecules are used for the treatment of Candida infections when the traditional drugs become registrant to these infections. Different molecules/substances acts to different infections having some side effects. Useful component of the plant are present in the leaves, seeds, fruits, and flowers. The concentration of natural compound extract depends on the chemical used for extraction and the storage conditions (Martins et al., 2015). Dichloromethane, methanol, ethanol, ethyl acetate, and n-butanol are some examples of chemical used for extraction process. There are many plants which have antimicrobial activity. One example is Lonicera japonica, a medicinal plant of China is used to treat various microbial activities. It is active against $C$. albicans and $C$. tropicalis and capable to heal the wounds (Chen et al., 2012).

Lannea welwitschi is discovered by Agyare et al. (2013). It is a medicinal plant active against $C$. albicans. Antimicrobial compound are mainly present in the leaves of Lannea welwitschi extracted by the solvent methanol. Main antimicrobial compound of Lannea welwitschi are flavonoid, alkaloids, tannis. Anti-candidal activities are also observed in the Pyrostegia venusta's flower extract (Pereira et al., 2014) and leaves of piper betel (Nordin et al., 2014). These different polyphenols which are susceptible to Candida infections are divided into two parts that are planktonic MIC (PMIC) and sessile MIC (SMIC).

Leave extract from Juglans regia and Eucalyptus globules are highly active against all the Candida strains (Martins et al., 2015). Acetylic extract of Vicia faba showed excellent activity against $C$. albicans having very low MIC value $(0.010 \mathrm{mg} / \mathrm{ml})$ and when experimented to mice it reduce the mortality rate with lower concentration of doses. Carya illinoensis have phytochemicals present in their leaves which show antimicrobial activity. Aqueous and ethanolic extract of Carya illinoensis is useful in the inhibition of production of germ tubes of $C$. albicans. Organic compounds, having low molecule weight polyphenols are mostly found in plants (Shahzad et al., 2014).

Flavonoids, tannins, phenolic acid are example of polyphenols which are used for different treatments as they act as anticancer, anti allergen antioxidants and antimicrobial agents. Flavonoids and tannins are mostly known for their antimicrobial activity. Flavonoids are highly active against $C$. albicans by the method of agar micro dilutions (Herrera et al., 2010). Tannins are isolated from the different parts of the plant such as fruit, leaves, flower, seed, and fruits. Elagitannins, proanthocyaninidins, and gallotanninas are the subclasses of tannins (Duval and Averous, 2016).

Mechanism of action of tannins is different against the different microorganism and not clear. Some essential oils from aromatic medicinal plant are also active against different microbes. Tea tree oil is important as it is active against Candida spp. which is multidrug-resistant especially in case of mucosal Candidiasis (Mondello et al., 2013). A combination of cuminlim cyminum and essential oil is highly susceptible to C. albicans and MIC value ranges from 3.9-11.71. Main advantage of essential oil is that they are able to inhibit the biofilms formation by inhibiting the formation of germination tube in C. albicans and at lower concentration value, i.e., 0.16. Example is Artemisia juduica (Abu-Darwish et al., 2016). C. albicans which are resistant to fluconazole show susceptibility to the essential oils extracted from Trachyspermum ammi.

Terpenoids are highly susceptible and show excellent activity against blastopores and filamentous form growth of $C$. albicans. Terpenes are useful to prevent the biofilms formation and its mechanism of action is simply the alteration in cytoplasmic membrane leading to apoptosis. Another naturally occurring compound which has anti Candida properties is phenylpropanoids. They are divided into coumarins phenylpropanoids acid and ligans (Lu et al., 2017). Coumarins are also designated as scopoletin. Scopoletins are susceptible against $C$. albicans having MIC value $25 \mu \mathrm{g} / \mathrm{mg}$ (Navarro et al., 2011). As these different compounds are used in combination the main advantage is that the lower doses of antifungal agents are required. Along with this there is less development of drug resistance. Other important approach to fight with Candida infection is immunotherapeutic approach. Some of immunotherapeutic approaches include vaccination, therapeutic antibodies, recombinant cytokines, and adoptive transfer of primed immune cells.

\subsection{Vaccination}

There is no vaccine against Candida infection has been developed but there are some other vaccines are present which can be used to treat Candida infections. One example of such vaccine is diphtheria toxoid CRM 197 conjugated with algal antigen luminarian and 
encouraging positive results have been seen in animal model (Tobudic et al., 2011). But, the main challenge is to provide immunity to immunocompromised patients as the vaccination is dependent on appropriate host mechanism to provide protection. Recombinant vaccines are produced by using adjuvants as these adjuvants are able to give shape in to the kind of adaptive immune response obtained by a vaccine. This can be done by producing different cytokines profiles (Ellis et al., 2002; Mata-Haro et al., 2007).

Cholera toxin is one example of adjuvant used. These changes are used to produce defense mechanism against the specific pathogen. For example in case of mucosal candidiasis adjuvant should aid a strong th17. Vaccine for $C$. albicans has successfully completed phase one clinical trials and the vaccine is univalent subunit vaccine. If approved, it will be the first antifungal vaccine for human use. A problem of achieving vaccine is that $C$. albicans are able to deceive the host immune system (Table 4).

\subsection{Antibodies}

To prevent or treat Candida infection another immunotherapeutic approach is using anti Candida antibodies. This approach is helpful in the immunocompromised patient (Table 4). Antibodies produced in animal models and patients with Candidiasis are artificially induced to the body. One example of human recombinant antibody is Efungumab. Efungumab works against $\mathrm{Hsp} 90$ which is a fungal specific protein. This antibody is not given solely but in the combination with different drugs like amphotericin B and caspofungin. When used in combination positive outcomes have noted. Including this monoclonal antibodies and immune serum from vaccinated mice is another immunotherapy to treat Candida infections. Mice are vaccinated with Candida mannans which have liposomes, anti-mannan antibodies, protective antibodies, and anti$\beta$ glucan antibodies (Veerdank et al., 2010). They are able to degrade the effect of toxins produced by yeast cells (Polonelli et al., 1999).

\subsection{Cytokines therapy}

Regulation of immunological responses during infection is done by cytokines. Cytokines are the signaling molecules that aid cell to cell communication in immune responses. Glycoprotein secreted by macrophages known as CSF or granulocyte-macrophage colonystimulating factor (GM-CSF). GM-CSF is responsible for increased phagocytosis and release of reactive oxygen species (Table 4). This is achieved by prolonged survival of neutrophils, unregulated expression of dectin-1 on macrophage and clearing chitin of cell wall of $C$. albicans (Van Eijk et al., 2005; Veerdonk et al., 2010). GM-CSF is able to decrease the fungal growth in humans and it can be used as an adjuvant for mycological improvements (Kullberg et al., 2005). Due to increase in macrophage cytotoxicity caused by IFN¥, intracellular pathogens are killed (Hubel et al., 2002). Patients suffering from granulomatous disease are most likely treated with IFN¥ for Candida infections (Baddley et al., 2008).

Table 4: Strategies for management of C. albicans infection (Veerdonk et al. 2010).

\begin{tabular}{|c|c|c|}
\hline Novel strategies & Advantages & Disadvantages \\
\hline Vaccination & $\begin{array}{l}\text { Broad spectrum activity } \\
\text { Low risk of the development of resistance } \\
\text { Long term effects. }\end{array}$ & $\begin{array}{l}\text { Dependent on the competence of host immune } \\
\text { status } \\
\text { Most patients with disseminated candidiasis are } \\
\text { immunocompromised might also induce disease } \\
\text { enhancing Ab relatively slow effects. }\end{array}$ \\
\hline Antibodies ( $\mathrm{Ab}$ ) & $\begin{array}{l}\text { Can be directly fungicidal independent of host } \\
\text { immune status. Very specific (e.g., strain specific) }\end{array}$ & $\begin{array}{l}\text { May induce the development of anti anti-bodies. } \\
\text { potentially toxic }\end{array}$ \\
\hline Cytokines & $\begin{array}{l}\text { Efficacy and safety in patients. Rapid effects are } \\
\text { seen. }\end{array}$ & $\begin{array}{l}\text { Mainly effective as adjuvant therapy. Might } \\
\text { influence the pharmacokinetics and efficacy } \\
\text { of the combined antifungal drugs.Inflammation } \\
\text { as a potential consequence. }\end{array}$ \\
\hline $\begin{array}{l}\text { Adoptive transfer } \\
\text { of primer immune } \\
\text { cells }\end{array}$ & $\begin{array}{l}\text { Opportunity to develop immune cells before } \\
\text { immunocompromised patients. }\end{array}$ & $\begin{array}{l}\text { Risk for developing graft-versus-host disease } \\
\text { and transplantation. }\end{array}$ \\
\hline
\end{tabular}

\section{Conclusion}

C. albicans has been of profound interest to the scientific community for its diverse pathogenic nature. When compared with $S$. cerevisiae, it seems that it is more complex. $80 \%$ genes of $C$. albicans found similar to $S$. cerevisiae. It is mostly found in immunocompromised patients. It can be treated by different drugs using different mechanisms, and these different drugs can be resisted by $C$. albicans through different mechanisms. Mechanistic studies have provided valuable insights into the regulatory circuitry and network that cover biofilm formation. These studies have revealed new mechanisms and signal that govern C. albicans biofilm formation are associated with drug resistance providing therapeutic foresight. Resistance of $C$. albicans to drugs can be prevented by giving higher doses of drugs for longer duration. If higher doses are not able to cure the Candida infection another technique is using different drugs in a proper composition. By understanding its pathogenecity mechanism, new antifungal therapies are introduced.

\section{Future perspectives}

Due to different resistance mechanism, it is important to know its proper mechanism of resistance and how it interacts with drugs. Another factor is finding some other sites of Candida which can be attacked by different drugs to kill or inhibit the growth of $C$. albicans. Vaccine should be provided against $C$. albicans specifically. Carbon utilization process helps to understand how it contributed to host fungal infection that reveals important virulence regulate that may be from promising antifungal target. Innate immunity system and antimicrobial activity of phagocytosis clean pathogen but $C$. albicans is able to survive within macrophage phagosome. So, it is an 
interesting fact to understand the intimate host pathogen interaction that occurs between macrophages and C. albicans. Several Candida pathogens up regulate alternative carbon utilization pathways upon phagocytosis suggesting that the connection between metabolism and pathogenesis is conserved pathways. Future studies will focus on importance of alternative carbon metabolism in related species especially the multidrug resistance emerging pathogens $C$. auris. To overcome the disadvantages of classical drugs available, new drugs should be introduced which works on different mechanism or by acting on different genes as compared to classical one.

\section{Acknowledgements}

Authors acknowledge the facilities provided by Department of Biotechnology, Himachal Pradesh University, Shimla.

\section{Conflict of interest}

The authors declare no conflicts of interest relevant to this article.

\section{References}

Abu-Darwish, M.; Cabral, C.; Gonçalves, M.; Cavaleiro, C.; Cruz, M.; Zulfiqar,A.; Khan, I.; Efferth, T. and Salgueiro, L. (2016). Chemical composition and biological activities of Artemisia judaica essential oil from southern desert of Jordan. J. Ethnopharmacol., 191:161-168.

Alexander, B.; Johnson, M.; Pfeiffer, C.; Jiménez-Ortigosa, C.; Catania, J.; Booker, R.; Castanheira, M.; Messer, S.; Perlin, D. and Pfaller, M. (2013). Increasing echinocandin resistance in candida glabrata: Clinical failure correlates with presence of FKS mutations and elevated minimum inhibitory concentrations. Clin. Infectious Dis., 56:1724-1732.

Arendrup, M. and Patterson, T. (2017). Multidrug-Resistant Candida: epidemiology, molecular mechanisms, and treatment. J. Infectious Dis., 216:S445-S451.

Baddley, J.; Patel, M.; Bhavnani, S.; Moser, S. and Andes, D. (2008). Association of fluconazole pharmacodynamics with mortality in patients with candidemia. Antimicrob. Agents Chemother., 52:3022-3028.

Balashov, S.; Park, S. and Perlin, D. (2006). Assessing resistance to the echinocandin antifungal drug caspofungin in Candida albicans by profiling mutations in FKS1. Antimicrob. Agents Chemother., 50: 2058-2063

Barwicz, J.; Dumont, I.; Ouellet, C. and Gruda, I. (1998). Amphotericin B toxicity as related to the formation of oxidatively modified low-density lipoproteins. Biospectroscopy, 4: 135-144.

Blumberg, H.; Jarvis, W.; Soucie, J.; Edwards, J.; Patterson, J.; Pfaller, M.; Rangel Frausto, M.; Rinaldi, M.; Saiman, L.; Wiblin, R. and Wenzel, R. (2001). Risk factors for candidal bloodstream infections in surgical intensive care unit patients: The NEMIS prospective multicenter study. Clinic. Infectious Dis., 33:177-186.

Bossche, H;, Willemsens, G. and Marichal, P. (1987). Anti-candida drugs: The biochemical basis for their activity. CRC Critic. Rev. Microbiol. 15:57-72.

Calderone, R.; Odds, F. and Boekhout, T. (2009). Candida albicans: Fundamental research on an opportunistic human pathogen. FEMS Yeast Res., 9:971-972.

Cannon, R.; Lamping, E.; Holmes, A.; Niimi, K.; Baret, P.; Keniya, M.; Tanabe, K.; Niimi, M.; Goffeau, A. and Monk, B. (2009). Efflux-mediated antifungal drug resistance. Clinic. Microbiol. Rev., 22:291-321.

Cernika, J. and Subik, J. (2006). Resistance mechanisms in fluconazoleresistant Candida albicans isolates from vaginal candidiasis. Int. J. Antimicrob. Agents, 27:403-408.
Chen, W.; Liou, S.; Tzeng, T.; Lee, S. and Liu, I. (2012). Wound repair and antiinflammatory potential of Lonicera japonica in excision woundinduced rats. BMC Complementary Alternat. Med., 12:212-223.

Colombo, A.; Nucci, M.; Park, B.; Noueìr, S.; Arthington-Skaggs, B.; da Matta, D.; Warnock, D. and Morgan, J. (2006). Epidemiology of candidemia in Brazil: A nationwide sentinel surveillance of candidemia in eleven medical centers. J. Clinic. Microbiol., 44:2816-2823.

De-Oliveira Santos, G; Vasconcelos, C.; Lopes, A.; de-Sousa Cartágenes, M.; Filho, A.; de-Nascimento, F.; Ramos, R.; Pires, E.; de-Andrade, M.; Rocha, F. and deAndrade M. C. (2018). Candida infections and therapeutic strategies: Mechanisms of action for traditional and alternative agents. Frontiers Microbiol., 9:456-467.

De Sordi, L. and Mühlschlegel, F. (2009). Quorum sensing and fungal-bacterial interactions in Candida albicans: A communicative network regulating microbial coexistence and virulence. FEMS Yeast Res., 9:990-999.

Durack, D. (1995). Prevention of infective endocarditis. New Eng. J. Med., 332:38-44.

Duval, A. and Avérous, L. (2016). Characterization and physicochemical properties of condensed tannins from Acacia catechu. J. Agric. Food Chem., 64:1751-1760.

Eddins, J.; Jabra-Rizk, M.; Fallder, W. and Meiller, T. (2004). Fungal biofilms and drug resistance. J. Asso. Vascular Access, 9:166-167.

Ellis, D. (2002). Amphotericin B: Spectrum and resistance. J. Antimicrob. Chemother., 49:7-10.

Espinel-Ingroff, A. and Turnidge, J. (2016). The role of epidemiological cutoff values (ECVs/ECOFFs) in antifungal susceptibility testing and interpretation for uncommon yeasts and moulds. Revista Iberoamericana de Micología, 33:63-75.

Eyerich, K.; Eyerich, S.; Hiller, J.; Behrendt, H. and Traidl-Hoffmann, C. (2010). Chronic mucocutaneous candidiasis, from bench to bedside. Eur. J. Dermatol., 20:260-265.

Franz, R.; Kelly, S.; Lamb, D.; Kelly, D.; Ruhnke, M. and Morschhauser, J. (1998). Multiple molecular mechanisms contribute to a stepwise development of fluconazole resistance in clinical Candida albicans strains. Antimicrob. Agents Chemother., 42:3065-3072.

Groll, A. (1997). Fluconazole versus nystatin in the prevention of Candida infections in children and adolescents undergoing remission induction or consolidation chemotherapy for cancer. J. Antimicrob. Chemother., 40:855-862.

Hakki, M.; Staab, J. and Marr, K. (2006). Emergence of a Candida krusei isolate with reduced susceptibility to caspofungin during therapy. Antimicrob. Agents Chemother., 50:2522-2524.

Henry, K.; Nickels, J. and Edlind, T. (2000). Upregulation of ERG genes in Candida species by azoles and other sterol biosynthesis inhibitors. Antimicrob. Agents Chemother., 44:2693-2700.

Hernandez, S.; Loìpez-Ribot, J.; Najvar, L.; McCarthy, D.; Bocanegra, R. and Graybill, J. (2004). Caspofungin resistance in Candida albicans: correlating clinical outcome with laboratory susceptibility testing of three isogenic isolates serially obtained from a patient with progressive Candida esophagitis. Antimicrob. Agents., 48:13821383.

Herrera, C.; Alvear, M.; Barrientos, L.; Montenegro, G. and Salazar, L. (2010). The antifungal effect of six commercial extracts of Chilean propolis on Candida spp. Ciencia e Investigación Agraria, 37:75-84.

Hoffman, H.; Ernst, E. and Klepser, M. (2000). Novel triazole antifungal agents. Expert Opinion Investigational Drugs, 9:593-605. 
Hübel, K.; Dale, D. and Liles, W. (2002). Therapeutic use of cytokines to modulate phagocyte function for the treatment of infectious diseases: current status of granulocyte colony stimulating factor, granulocyte macrophage colony stimulating factor, macrophage colony stimulating factor, and interferon $\gamma$. J. Infect. Dis., 185: 1490-1501.

Huppler, A.; Bishu, S. and Gaffen, S. (2012). Mucocutaneous candidiasis: The IL-17 pathway and implications for targeted immunotherapy. Arthritis Res. Ther., 14:217-229.

Johnson, E.; Davey, K.; Szekely, A. and Warnock, D. (1995.) Itraconazole susceptibilities of fluconazole susceptible and resistant isolates of five Candida species. J. Antimicrob. Chemother., 36:787-793.

Kabir, M.; Hussain, M. and Ahmad, Z. (2012). Candida albicans: A model organism for studying fungal pathogens. ISRN Microbiol., 12;1-15.

Kahn, J.; Garcia-Effron, G.; Hsu, M.; Park, S.; Marr, K. and Perlin, D. (2007). Acquired echinocandin resistance in a Candida krusei isolate due to modification of glucan synthase. Antimicrob. Agents.Chemother. 51:1876-1878

Krishnakumari, V.; Rangaraj, N. and Nagaraj, R. (2009). Antifungal activitie of human beta-defensins HBD-1 to HBD-3 and their C-terminal analogs Phd1 to Phd3. Antimicrob. Agents Chemother., 53:256260.

Krogh-Madsen, M.; Arendrup, M.; Heslet, L. and Knudsen, J. (2006). Amphotericin $\mathrm{B}$ and caspofungin resistance in Candida glabrata isolates recovered from a critically ill patient. Clinic. Infect. Dis., 42:938-944.

Kuhn, D.; Chandra, J.; Mukherjee, P. and Ghannoum, M. (2002). Comparison of biofilms formed by Candida albicans and Candida parapsilosis on bioprosthetic surfaces. Infect. Immun., 70:878-888

Kullberg, B.; Sobel, J.; Ruhnke, M.; Pappas, P.; Viscoli, C.; Rex, J.; Cleary, J.; Rubinstein, E.; Church, L.; Brown, J.; Schlamm, H.; Oborska, I.; Hilton, F. and Hodges, M. (2005). Voriconazole versus a regimen of amphotericin B followed by fluconazole for candidaemia in non-neutropenic patients: a randomised non-inferiority trial. Lancet, 366:14351442

Livermore, D. (2004). The need for new antibiotics. Clinic. Microbiol. Infect., 10:1-9.

Lu, M.; Li, T.; Wan, J.; Li, X.; Yuan, L. and Sun, S. (2017). Antifungal effects of phytocompounds on Candida species alone and in combination with fluconazole. International J. Antimicrob. Agents, 49:125136

Lupetti, A.; Brouwer, C.; Bogaards, S.; Welling, M.; de Heer, E.; Campa, M.; van Dissel, J.; Friesen, R. and Nibbering, P. (2007). Human lactoferrin derived peptide's antifungal activities against disseminated Candida albicans infection. J. Infectious Dis., 196:1416-1424.

Marichal, P.; Vanden Bossche, H.; Odds, F.; Nobels, G.; Warnock, D.; Timmerman, V.; Van Broeckhoven, C.; Fay, S. and Mose-Larsen, P. (1997). Molecular biological characterization of an azole-resistant Candida glabrata isolate. Antimicrob. Agents Chemother., 41:2229-2237.

Marr, K.; Lyons, C.; Rustad, T.; Bowden, R. and White, T. (1998). Rapid, transient fluconazole resistance in Candida albicans is associated with increased mRNA levels of CDR. Antimicrob. Agents Chemother., 42:2584-2589.

Martins, N.; Barros, L.; Henriques, M.; Silva, S. and Ferreira, I. (2015). In vivo anti-candidaactivity of phenolic extracts and compounds: Future perspectives focusing on effective clinical interventions. Bio. Med. Res. Int., pp:1-14.

Mata-Haro, V.; Cekic, C.; Martin, M.; Chilton, P.; Casella, C. and Mitchell, T. (2007). The vaccine adjuvant monophosphoryl lipid A as a TRIF-biased agonist of TLR4. Science, 316:1628-1632.
Meis, J. and Verweij, P. (2001). Current management of fungal infections. Drugs, 61:13-25.

Mondello, F.; De Bernardis, F.; Girolamo, A.; Cassone, A. and Salvatore, G. (2006) In vivo activity of terpinen-4-ol, the main bioactive component of Melaleuca alternifolia Cheel (tea tree) oil against azolesusceptible and -resistant human pathogenic Candida species. BMC Infectious Dis., 6:1223-1229.

Morace, G.; Perdoni, F. and Borghi, E. (2014). Antifungal drug resistance in Candida species. J. Global Antimicrob. Resistance, 2:254-259.

Morici, P.;Fais, R., Rizzato, C.; Tavanti, A. and Lupetti, A. (2016). Inhibition of Candida albicans biofilm formation by the synthetic lactoferricin derived Peptide hLF1-11. PLOS ONE, 11:e0167470.

Navarro-García, V.; Rojas, G.; Avilés, M.; Fuentes, M. and Zepeda, G. (2011). In vitro antifungal activity of coumarin extracted from Loeselia mexicana brand. Mycoses, 54:e569-e571.

Nordin, M.; Wan Harun, W.; Abdul Razak, F. and Musa, M. (2014). Growth inhibitory response and ultrastructural modification of oralassociated candidal reference strains (ATCC) by Piper betle L. extract. Int. J. Oral Sci., 6:15-21.

Odds, F. (1987). Candida infections: an overview. CRC Crit. Rev. Microbiol., 15: $1-5$

Onyewu, C. and Heitman, J. (2007). Unique applications of novel antifungal drug combinations. Anti-Infective Agents Medic. Chem., 6:3-15.

Orsi, N. (2004). The antimicrobial activity of lactoferrin: Current status and perspectives. Bio. Metals, 17:189-196.

Pappas, P.; Kauffman, C.; Andes, D.; Clancy, C.; Marr, K.; Ostrosky-Zeichner, L.; Reboli,A.; Schuster, M.; Vazquez, J.; Walsh, T.; Zaoutis, T. and Sobel, J. (2015). Clinical practice guideline for the management of candidiasis: 2016 update by the Infectious Diseases Society of America. Clinic. Infectious Dis., 62:e1-e50.

Park, S.; Kelly, R.; Kahn, J.; Robles, J.; Hsu, M.; Register, E.; Li, W.; Vyas, V.; Fan, H.; Abruzzo, G; Flattery,A.; Gill, C.; Chrebet, G.; Parent, S.; Kurtz, M.; Teppler, H.; Douglas, C. and Perlin, D. (2005). Specific substitutions in the echinocandin target Fks1p account for reduced susceptibility of rare laboratory and clinical Candida sp. isolates. Antimicrob. Agents Chemother., 49:3264-3273.

Pasqualotto, A. and Denning, D. (2008). New and emerging treatments for fungal infections. J. Antimicrob. Chemother., 61:i19-i30.

Pemán, J.; Cantón, E. and Espinel-Ingroff, A. (2009). Antifungal drug resistance mechanisms. Expert Rev. Anti-infective Ther., 7:453-460.

Perea, S.; Loìpez-Ribot, J.; Wickes, B.; Kirkpatrick, W.; Dib, O.; Bachmann, S.; Keller, S.; Martinez, M. and Patterson, T. (2002). Molecular Mechanisms of fluconazole resistance in Candida dubliniensis isolates from human immunodeficiency virus-infected patients with oropharyn-geal candidiasis. Antimicrob. Agents Chemother., 46:1695-1703.

Pereira, A.; Hernandes, C.; Pereira, S.; Bertoni, B.; França, S.; Pereira, P. and TalebContini, S. (2014). Evaluation of anticandidal and antioxidant activities of phenolic compounds from Pyrostegia venusta (Ker Gawl.) Miers. Chemico-Biological Interactions, 224:136-141.

Pfaller, M.; Messer, S.; Boyken, L.; Rice, C.; Tendolkar, S.; Hollis, R. and Diekema, D. (2004). Cross-resistance between fluconazole and ravuconazole and the use of fluconazole as a surrogate marker to predict susceptibility and resistance to ravuconazole among 12,796 clinical isolates of Candida spp. J. Clinic. Microbiol., 42:3137-3141.

Pfaller, M.; Messer, S.; Boyken, L.; Rice, C.; Tendolkar, S.; Hollis, R. and Diekema, D. (2007). Use of fluconazole as a surrogate marker to predict susceptibility and resistance to voriconazole among 13,338 clinical isolates of Candida spp. tested by clinical and laboratory standards institute-recommended broth microdilution methods. J. Clinic. Microbiol., 45:70-75. 
Polonelli, L. and Cassone, A. (1999). Novel strategies for treating candidiasis. Curr. Opin. Infectious Dis., 12:61-66.

Redding, S.; Kirkpatrick, W.; Saville, S.; Coco, B.; White, W.; Fothergill, A.; Rinaldi, M.; Eng, T.; Patterson, T. and Lopez-Ribot, J. (2003). Multiple patterns of resistance to fluconazole in Candida glabrata isolates from a patient with oropharyngeal candidiasis receiving head and neck radiation. J. Clinic. Microbiol., 41:619-622.

Rex, J.; Walsh, T.; Sobel, J.; Filler, S.; Pappas, P.; Dismukes, W. and Edwards, J. (2000). Practice guidelines for the treatment of candidiasis. Clinic. Infectious Dis., 30:662-678.

Ribeiro, M.; Paula, C.; Perfect, J. and Cox, G. (2005). Phenotypic and genotypic evaluation of fluconazole resistance in vaginal Candida strains isolated from HIV-infected women from Brazil. Medical Mycol., 43:647-650.

Riley, V.; Odds, F.; Fisk, P. and Webster, C. (1986). Finger nails as a reservoir for Candida albicans in recurrence of vaginal candidosis. Sexually Transmitted Infect., 62:358-358.

Rodrigues, M.; Silva, S.; Azeredo, J. and Henriques, M. (2014). Novel strategies to fight Candida species infection. Crit. Rev. Microbiol., 42:594606

Rogers, P. and Barker, K. (2003). Genome-wide expression profile analysis reveals coordinately regulated genes associated with stepwise acquisition of azole resistance in Candida albicans clinical isolates. Antimicrob. Agents Chemother., 47:1220-1227.

Ruhnke, M.; Rickerts, V.; Cornely, O.; Buchheidt, D.; Glöckner, A.; Heinz, W.; Höhl, R.; Horré, R.; Karthaus, M.; Kujath, P.; Willinger, B.; Presterl, E.; Rath, P.; Ritter, J.; Glasmacher, A.; Lass-Flörl, C. and Groll, A (2011). Diagnosis and therapy of Candida infections: Joint recommendations of the German Speaking Mycological Society and the Paul-Ehrlich-Society for Chemotherapy. Mycoses, 54:279-310.

Safdar, A.; van Rhee, F.; Henslee-Downey, J.; Singhal, S. and Mehta, J. (2001). Candida glabrata and Candida krusei fungemia after high-risk allogeneic marrow transplantation: no adverse effect of low-dose fluconazole prophylaxis on incidence and outcome. Bone Marrow Transplantation, 28:873-878.

Samaranayake, Y.; Samaranayake, L.; Wu, P. and SO, M. (1997). The antifungal effect of lactoferrin and lysozyme on Candida krusei and Candida albicans. APMIS, 105:875-883.

Sanglard, D.; Ischer, F.; Monod, M. and Bille, J. (1996). Susceptibilities of Candida albicans multidrug transporter mutants to various antifungal agents and other metabolic inhibitors. Antimicrob. Agents Chemother., 40:2300-2305.
Seleem, D.; Pardi, V. and Murata, R. (2017). Review of flavonoids: A diverse group of natural compounds with anti-Candida albicans activity in vitro. Arch. Oral Biol., 76:76-83.

Shahzad, M.; Sherry, L.; Rajendran, R.; Edwards, C.; Combet, E. and Ramage, G. (2014). Utilising polyphenols for the clinical management of Candida albicans biofilms. Int. J. Antimicrob. Agents, 44:269-273.

Skiest, D.; Vazquez, J.; Anstead, G.; Graybill, J.; Reynes, J.; Ward, D.; Hare, R.; Boparai, N. and Isaacs, R. (2007). Posaconazole for the treatment of azole-refractory oropharyngeal and esophageal candidiasis in subjects with HIV infection. Clinic. Infectious Dis., 44:607-614.

Spampinato, C. and Leonardi, D. (2013). Candida infections, causes, targets, and resistance mechanisms: Traditional and alternative antifungal agents. BioMed Res. International, 12:1-13.

Tobudic, S.; Kratzer, C;, Lassnigg, A. and Presterl, E. (2011). Antifungal susceptibility of Candida albicans in biofilms. Mycoses, 55:199-204.

Tomalka, J.; Azodi, E.; Narra, H.; Patel, K.; O’Neill, S.; Cardwell, C.; Hall, B.; Wilson, J. and Hise, A. (2015). $\beta$-Defensin 1 plays a role in acute mucosal defense against Candida albicans. J. Immunol., 194:1788-1795.

Van De Veerdonk, F.; Netea, M.; Joosten, L.; Van Der Meer,J. and Kullberg, B. (2010). Novel strategies for the prevention and treatment of Candida infections: the potential of immunotherapy. FEMS Microbiol. Rev., 34:1063-1075

van der Does, A.; Joosten, S.; Vroomans, E.; Bogaards, S.; van Meijgaarden, K.; Ottenhoff, T.; van Dissel, J. and Nibbering, P. (2012). The antimicrobial peptide hlf1-11 drives monocyte-dendritic cell differentiation toward dendritic cells that promote antifungal responses and enhance Th17 polarization. J. Innate Immun., 4:284-292.

Van-Eijk, M.; van-Roomen, C.; Renkema, G.; Bussink, A.; Andrews, L.; Blommaart, E.; Sugar, A.; Verhoeven, A.; Boot, R. and Aerts, J. (2005). Characterization of human phagocyte-derived chitotriosidase, a component of innate immunity. Int. Immunol., 17:1505-1512.

Vazquez, J.; Peng, G.; Sobel, J.; Steele Moore, L; Schuman, P.; Holloway, W. and Neaton, J. (2001). Evolution of antifungal susceptibility amongcandidaspecies isolates recovered from human immunodeficiency virus-infected women receiving fluconazole prophylaxis. Clinic. Infectious Dis., 33:1069-1075.

White, T.; Marr, K. and Bowden, R. (1998). Clinical, cellular, and molecular factors that contribute to antifungal drug resistance. Clinic. Microbiol. Rev., 11:382-402.

Williams, D.; Kuriyama, T.; Silva, S.; Malic, S. and Lewis, M. (2010). Candida biofilms and oral candidosis: treatment and prevention. Periodontol., 55:250-265. 